\title{
Anionic surfactant induced desorption of a cationic surfactant from mica
}

\author{
Finian J. Allen ${ }^{\mathrm{a}}$, Chris L. Truscott ${ }^{\mathrm{a}}$, Rebecca J. L. Welbourn ${ }^{\mathrm{b}}$, Stuart M. \\ Clarke $^{\mathrm{a}, *}$ \\ ${ }^{a}$ Department of Chemistry and BP Institute, University of Cambridge, Cambridge, UK \\ ${ }^{b}$ ISIS Pulsed Neutron Facility, Harwell Science and Innovation Campus, STFC, Rutherford \\ Appleton Laboratory, Didcot, Oxon, UK
}

\begin{abstract}
The adsorption and desorption of a cationic surfactant, didodecyldimethylammonium bromide, from water onto a mica surface has been investigated using neutron reflectivity. The surfactant was observed to adsorb strongly as a bilayer that was tenacious to a sustained water wash, but on the addition of an anionic surfactant, sodium dodecyl sulfate, at its critical micelle concentration complete desorption was observed.
\end{abstract}

Keywords:

Neutron reflectivity, Mica, Didodecyldimethylammonium bromide, Sodium dodecyl sulfate, Surfactant, Catanionic

\section{Introduction}

The interaction between clays and surfactants is of relevance to numerous fields, from surfactant based enhanced oil recovery (EOR) (Sheng, 2015), to organoclays, which are frequently synthesised from quaternary alkylammonium compounds (de Paiva et al., 2008). The behaviour of these surfactants at the clay surfaces is challenging to experimentally determine. Muscovite mica is often used as a model clay surface due to the perfect basal cleavage the crystal

\footnotetext{
* Corresponding author

Email addresses: fa316@cam.ac.uk (Finian J. Allen), clt45@cam.ac.uk (Chris L. Truscott), becky.welbourn@stfc.ac.uk (Rebecca J. L. Welbourn), stuart@bpi.cam.ac.uk (Stuart M. Clarke)
}

Preprint submitted to Applied Clay Science

February 5, 2018 
undergoes, which allows flat and clean surfaces to be easily generated. Muscovite is a phyllosilicate mineral with isomorphic substitution of one $\mathrm{Si}^{4+}$ by $\mathrm{Al}^{3+}$ per unit cell. This structural charge is compensated for by interlayer $\mathrm{K}^{+}$ions, giving an idealised formula of $\mathrm{KAl}_{2}\left(\mathrm{AlSi}_{3} \mathrm{O}_{10}\right)(\mathrm{OH})_{2}$ and an area of $47 \AA^{2}$ per charge site (Schlegel et al., 2006). As this charge is structural, little $\mathrm{pH}$ dependence of the mica surface charge is expected or observed (Zhao et al. 2008). The use of 'bulk' techniques to observe behaviour of mica and other clays requires a powder to be used to give sufficient surface area for analysis. This complicates measurements of the basal surface due to the vastly increased contribution of edge site behaviour and properties which are different in most aspects to the basal plane. The number of techniques which can be used to observe basal plane behaviour is therefore limited.

A variety of techniques are used to investigate the solid/liquid interface though typically these are 'invasive' techniques where a second surface is introduced in order to make the measurement. Examples of these are atomic force microscopy (AFM), and the surface force apparatus (SFA) (Perkin, 2012). There is evidence that the 'frontal confinement' caused by the second surface can perturb the system and result in non-equilibrium structures being measured (Speranza et al., 2013, Striolo and Grady, 2017).

Neutron reflectometry is a well established, and non-invasive technique that allows behaviour at buried interfaces to be measured. In addition the use of contrast variation, exchanging hydrogen for deuterium to change the scattering of different components while maintaining the chemistry, can allow simultaneous fitting of multiple datasets for a system (Sivia, 2011). Substrate preparation is challenging as an extremely flat surface is required over a large area (the order of $\mathrm{cm}^{2}$ ) and, as the beam passes through the solid to reach the solid liquid interface, substrate attenuation of the beam must be minimised. Both of these factors present particular issues for the mineral mica, which shows crystal waviness if unsupported and attenuates strongly.

In recent work, Browning et al. (2014) showed that by using a silicon wafer to support a thin mica sheet, neutron reflectivity (NR) could be successfully 
applied to the mica/solution interface. This set up has since been applied successfully to numerous studies (Allen et al., 2017; Griffin et al., 2016) and has previously been used to investigate adsorption of didodecyldimethylammonium bromide (DDAB) adsorption to the mica basal plane (Browning et al., 2014 Griffin et al., 2016). The layer was observed to adsorb as a bilayer of thickness $24 \pm 2 \AA$ and roughness $2 \pm 1 \AA$ in one case, and $23 \pm 2 \AA$ and roughness $2 \pm$ $1 \AA$ in the other, with effectively no water inclusion in the layer in both cases. These numbers are in good agreement with other measurements of DDAB bilayers on mica (Dubois and Zemb, 1991) and on quartz (Blom et al., 2007), and indicate an interdigitated or tilted bilayer. It was observed that a wash with $\mathrm{D}_{2} \mathrm{O}$ caused the bilayer thickness to decrease to $20 \pm 1 \AA$. However subsequent washes with $10 \mathrm{mM} \mathrm{KCl}$ or $\mathrm{CaCl}_{2}$ did not alter the layer structure further. A $\mathrm{UV} /$ ozone treatment was required to remove the surfactant from the mica surface. In this work we probe the behaviour of sequential surfactant solutions at the mica surface rather than a single one-component solution as previously.

The interaction of cationic and anionic surfactants in solution has been the subject of much research, and mixed micelles termed catanionic micelles are known to form in solution (Sohrabi et al. 2008). Due to the favourable interactions the critical micelle concentration (CMC) of a cationic - anionic mixture would be expected to show a minimum at some concentration ratio with a surface tension below that of the CMC surface tension of either pure surfactant, and this has been observed experimentally (Holland and Rubingh, 1983).

There have been studies of the didodecyldimethylammonium bromide - sodium dodecyl sulfate (SDS) mixed system specifically; Marques et al. showed the pseudo-ternary phase diagram to be complex, containing regions of lamellar liquid crystalline phases, and regions of vesicles rich in the cationic or anionic surfactant depending on the molar ratio (Marques et al., 1993). The anionic rich region of the phase diagram has been studied further and for SDS solutions at the CMC of $0.24 \mathrm{wt} \%$, which is the concentration used in this work, it was observed that the solution was stable on addition of DDAB up to a mole fraction of 0.06 (Marques et al. 1999). Above this mole fraction precipitation occurred. 
CMC value were made up in $\mathrm{D}_{2} \mathrm{O}$, supplied by the ISIS neutron facility.

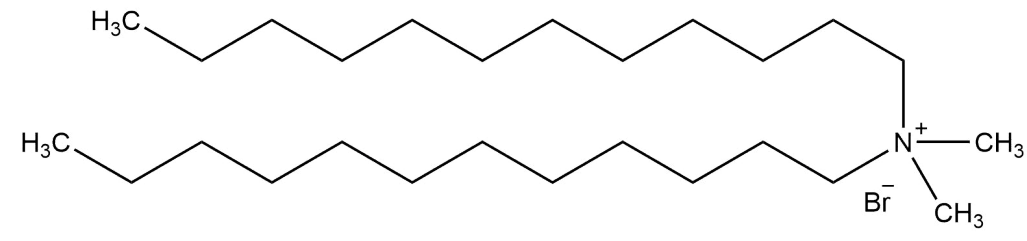

(a) Structure of DDAB, a di-chain cationic surfactant.

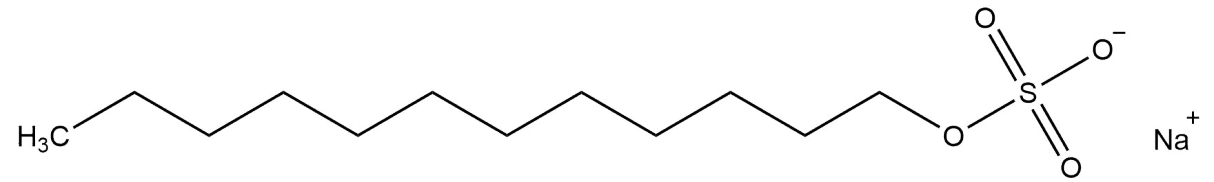

(b) Structure of SDS, an anionic mono-chain surfactant.

Figure 1: Structures of the surfactants used in this work.

is in agreement with work by Bai et al. who identified this region as con(Zhao et al., 2008). It is noted that these works were carried out in $\mathrm{H}_{2} \mathrm{O}$, and the use of $\mathrm{D}_{2} \mathrm{O}$, as in this study, has been seen to lead to small shifts in phase boundaries in similar systems (Kaler et al., 1992).

\section{Experimental}

\subsection{Materials}

Didodecyldimethylammonium bromide, DDAB, (Sigma, $\geq 98 \%$ ) and sodium dodecyl sulfate, SDS, (Sigma, $\geq 99 \%$ ) were used as received. The structures of the two surfactants are shown in Fig. 1. The CMC of DDAB has previously been determined for an identical batch of compound by surface tension measurements as $0.08 \mathrm{mM}$ and no minimum in the surface tension was observed (Browning et al. 2014). The CMC for SDS was taken from the literature to be $8.2 \mathrm{mM}$ Mukerjee and Mysels, 1971). Solutions of each surfactant at the respective 
with two $\mathrm{Al}$ atoms in the octahedral sheet per unit cell, and three Si atoms and one $\mathrm{Al}$ atom in the tetrahedral sheet. Relative to the atomic percentage of $\mathrm{K}$, the measured $\mathrm{K}: \mathrm{Al}$ : Si ratio was $1: 2.9: 3.5$, in reasonable agreement with the model formula. $\mathrm{Na}$ is a common impurity, replacing some of the exchangeable $\mathrm{K}$ ions, and $\mathrm{Fe}$ and $\mathrm{Mg}$ are common lattice impurities. $\mathrm{Na}, \mathrm{Fe}$, and $\mathrm{Mg}$ were all measured at less than 10 atomic\% of the measured K quantity in the sample. Neglecting impurities, the extent of tetrahedral isomorphic substitution may be estimated from assuming that there are two octahedral $\mathrm{Al}$ ions for each $\mathrm{K}$ (as mica is dioctahedral), and hence calculating the ratio of $\mathrm{Al}$ to $\mathrm{Si}$ in the tetrahedral sheet. This calculation gives a value of $21 \%$ of the tetrahedral sites filled by an $\mathrm{Al}$ substitution. This is again in reasonable agreement with the ideal structure, where $25 \%$ substitution is assumed.

\subsection{Substrate preparation}

Substrates for NR were prepared in the manner of Browning et al. (2014), with the modification of Griffin et al. (2015) to a larger substrate size, and is only briefly described here. A UV-curable glue (Loctite 3301) was spin coated onto a nitric acid cleaned neutron grade polished Si block (Crystran). A mica sheet was carefully cleaved, clamped against the glue layer using a highly polished Pyrex block (OptoSigma), and the glue was cured with a UV lamp (UVP CL1000, $\lambda=254 \mathrm{~nm})$. The mica was then cleaved again to yield a fresh surface which was UV/ozone cleaned and clamped against a teflon trough to form the sealed solid - liquid interface cell.

The cell was carefully filled to prevent trapped bubbles. Thereafter solutions were exchanged using a high performance HPLC pump. A volume of $30 \mathrm{ml}$ and flow rate of $2.0 \mathrm{ml} / \mathrm{min}$ was used for each solution change and washing steps to ensure complete exchange of the solution in the cell. This has previously been determined as a more than sufficient volume, using dye solutions to monitor exchange. The flow lines were flushed before and after each surfactant to avoid contamination. 


\subsection{Neutron reflectivity measurements}

Neutron reflectivity measurements were carried out at the INTER instrument at the ISIS neutron facility, UK (Webster et al., 2006), using time of flight to determine the wavelength of the incident neutron pulse. Three angles of incidence, $0.4^{\circ}, 0.9^{\circ}$, and $2.3^{\circ}$, were used to give access to the desired range of momentum transfer to the surface, $Q_{z}$, as defined in Equation 1 where $\lambda$ is the neutron wavelength and $\theta$ the angle of incidence.

$$
Q_{z}=\frac{4 \pi}{\lambda} \sin (\theta)
$$

\subsection{Data analysis}

NR data are analysed by modelling to a series of layers at the interface. Each layer is characterised by a thickness, roughness and scattering length density (SLD) which gives information about the composition.

The added complexity of the thick and thin layered substrate used in this work, compared to a more common NR substrate, results in the need for a different data analysis method. Both the mica and glue layers of the substrate are thicker than the coherence length of the neutrons, resulting in the loss of phase information as they pass through these layers. The more appropriate procedure is then to sum the intensity of the wave from these 'thick' layers, rather than the amplitude as is appropriate for 'thin' layers such as the $\mathrm{SiO}_{2}$ layer or an adsorbed surfactant layer. Data fitting was carried out using a modified version of the custom program I-CALC, described in more detail elsewhere (Browning et al. 2014), which deals appropriately with the thick and thin layers and also accounts for the wavelength and path-length dependent attenuation of the neutrons in the glue and mica.

The SLD is the volume average of the scattering lengths of the individual nuclei. SLDs for the materials may be calculated from an estimate of the molecular volume and the sum of the scattering lengths of the constituent atoms. The fitted SLD of a layer can be used to calculate the percentage of water inclusion, 
Table 1: Scattering Length Densities of Materials.

\begin{tabular}{lclc}
\hline Material & SLD $/ \times 10^{-6} \AA^{-2}$ & Material & SLD $/ \times 10^{-6} \AA^{-2}$ \\
\hline Silicon & 2.07 & $\mathrm{H}_{2} \mathrm{O}$ & -0.56 \\
Silicon oxide & 3.49 & $\mathrm{D}_{2} \mathrm{O}$ & 6.30 \\
Glue & 1.08 & $\mathrm{CMSi}^{\dagger}$ & 2.10 \\
Mica & 3.79 & $\mathrm{DDAB}^{\ddagger}$ & -0.25 \\
\hline
\end{tabular}

$\dagger$ CMSi refers to water contrast matched to the silicon substrate, to increase the scattering contribution from the mica. $\ddagger$ The SLD value for DDAB is that used by Browning et al. as calculated from volume considerations based on a density of 0.946 $\mathrm{g} \mathrm{cm}^{-3}$ from Grillo et al. (Grillo et al. 2009).

referred to as the hydration. The SLDs of materials used in this study are given in Table 1

\section{Results and Discussion}

\subsection{Results}

Fig. 2 shows the reflectivity data recorded from the bare mica surface in $\mathrm{H}_{2} \mathrm{O}, \mathrm{D}_{2} \mathrm{O}$, and an $\mathrm{H}_{2} \mathrm{O} / \mathrm{D}_{2} \mathrm{O}$ mix contrast matched to silicon (CMSi). The fits to the data result from the parameters given in Table 2, The SLD for the mica is calculated from the idealised formula and unit cell volume, and the close fits to the data generated from this parameter gives further confidence that the mica composition is close to this. The fitted roughness of the mica surface of 4 $\AA$ gives evidence for a good cleave, with very few step planes. The cell solution was then exchanged to DDAB at a concentration of $1 \mathrm{CMC}(0.08 \mathrm{mM})$ in $\mathrm{D}_{2} \mathrm{O}$, and the reflectivity profile recorded. This is shown in Fig. $3 \mathrm{a}$ in the lower profile. A clear change from the bare surface profile is observed, indicating adsorption of DDAB as expected. The fit to the data was calculated with the parameters in Table 2, and the fitted layer thickness of $22 \pm 1 \AA$ and lack of hydration are consistent with previous observations of a DDAB bilayer on mica where the layer thicknesses were $24 \pm 2 \AA$ and $23 \pm 2 \AA$, both with no hydration (Browning et al. 2014, Griffin et al., 2016). The thickness of a floating DDAB bilayer has been measured as $24 \AA$ (Dubois and Zemb, 1991). 
Subsequently the cell was washed through with $\mathrm{D}_{2} \mathrm{O}$. A slight change in profile is seen as shown in Fig. 3a in the upper profile, and the layer thickness decreases to $19 \pm 1 \AA$ still with no hydration. This is consistent with previous observations which found the DDAB layer decreased in thickness to $20 \pm 1 \AA$ after a water $/ \mathrm{D}_{2} \mathrm{O}$ wash or after washing with $10 \mathrm{mM} \mathrm{KCl}$ or $\mathrm{CaCl}_{2}$ Griffin et al., 2016).

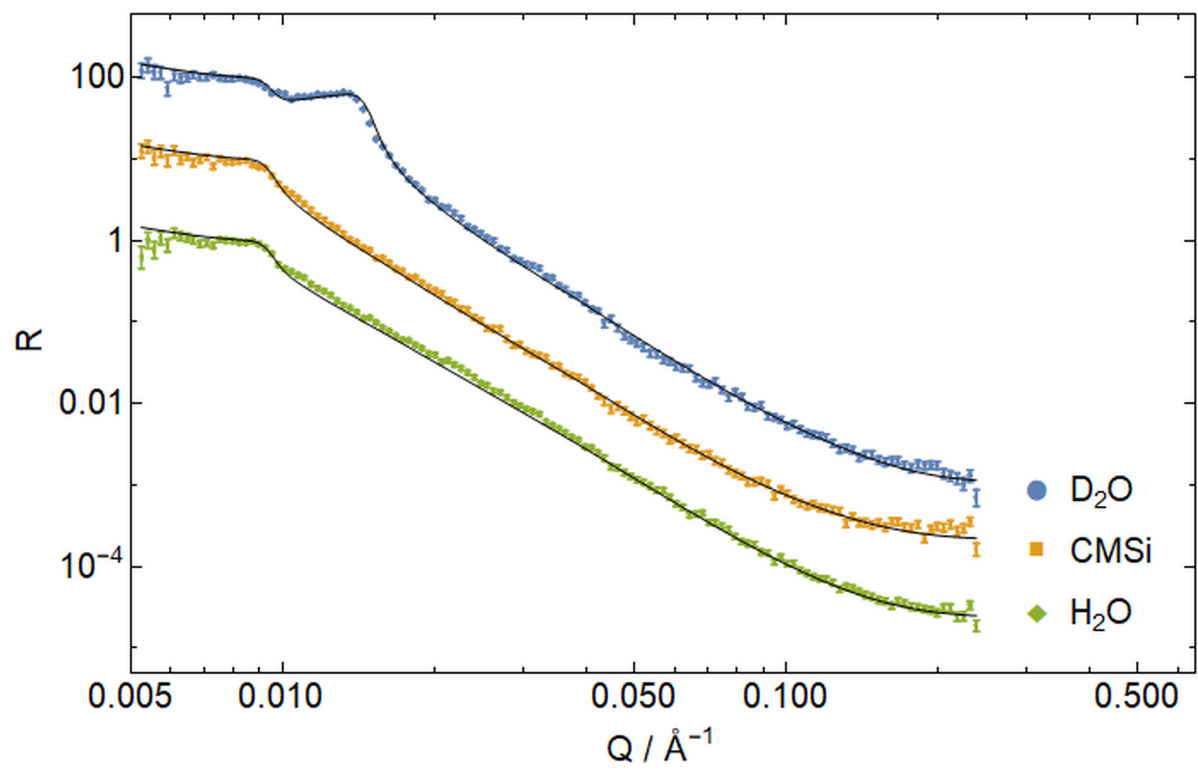

(a) Reflectivity profiles from the bare mica surface in various contrasts. Data are offset for clarity; CMSi data is offset by a factor of 10 upwards, and $\mathrm{D}_{2} \mathrm{O}$ offset by a factor of 100. Calculated fits are superimposed.

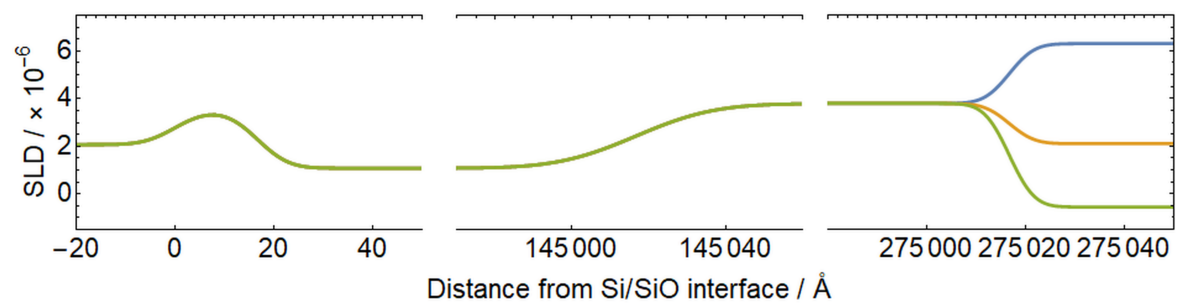

(b) Fitted SLD profile for the bare mica substrate, showing fits for $\mathrm{H}_{2} \mathrm{O}$ (bottom), CMSi (middle), and $\mathrm{D}_{2} \mathrm{O}$ (top).

Figure 2: Reflectivity data and fits for the bare mica substrate. 


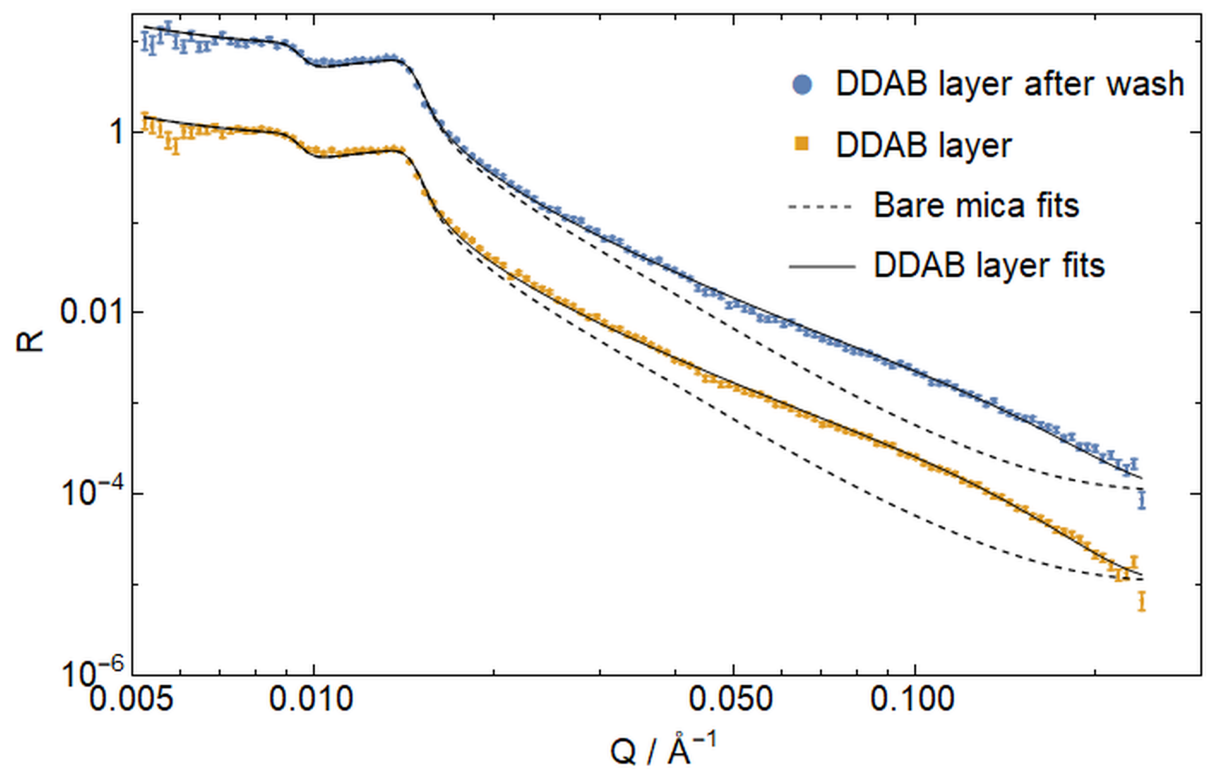

(a) Reflectivity profiles from mica substrate with $1 \mathrm{CMC}(0.08 \mathrm{mM})$ DDAB (lower profile) and following an extended $\mathrm{D}_{2} \mathrm{O}$ wash (upper profile, offset by a factor of 10). Dashed lines are the calculated fit for the bare mica, and solid lines are the two fits calculated for the DDAB layer.

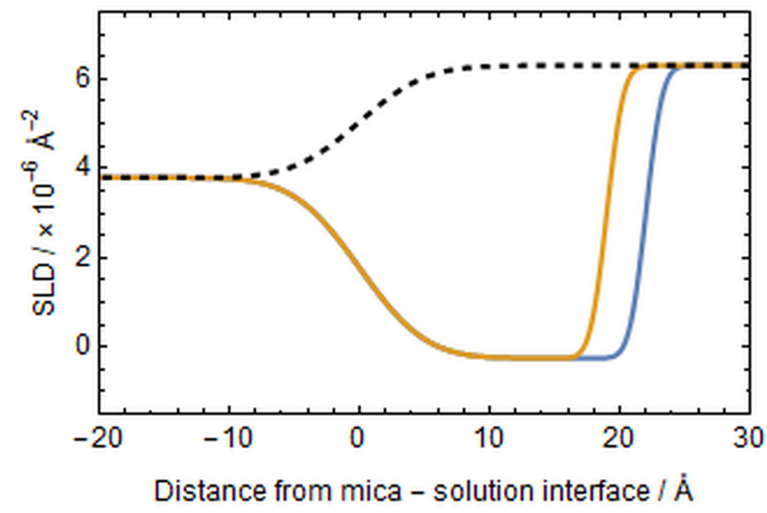

(b) SLD profiles fitted to the DDAB layers before (right line) and after (left line) a $\mathrm{D}_{2} \mathrm{O}$ wash. The dashed line is the SLD profile fitted to the bare mica.

Figure 3: Data and fits from the mica substrate after introduction of DDAB at 1 CMC $(0.08$ $\mathrm{mM}$ ) and after a subsequent $\mathrm{D}_{2} \mathrm{O}$ wash. The dashed black line is the SLD profile fitted to the bare surface.

Following the $\mathrm{D}_{2} \mathrm{O}$ wash, a solution of SDS in $\mathrm{D}_{2} \mathrm{O}$ at the SDS CMC value

171 (8.2 $\mathrm{mM})$ was exchanged into the cell. The reflectivity profile was remeasured, 
Table 2: Fitted parameters for bare surface and adsorbed layer.

\begin{tabular}{lccc}
\hline Material & Thickness & Roughness / & Hydration / \% \\
\hline Silicon & & 5 & \\
Silicon oxide & $16.7 \AA$ & 5 & \\
Glue & $14.5 \mu \mathrm{m}$ & 16 & \\
Mica & $13 \mu \mathrm{m}$ & 4 & \\
DDAB layer & $22 \pm 1 \AA$ & $1 \pm 1$ & $0 \pm 3$ \\
DDAB layer after $\mathrm{D}_{2} \mathrm{O}$ wash & $19 \pm 1 \AA$ & $1 \pm 1$ & $0 \pm 3$ \\
\hline
\end{tabular}

172

as shown in Fig. 4. The profile recorded after SDS introduction is identical to that of the bare mica substrate, revealing that the SDS solution has entirely removed the adsorbed DDAB layer.

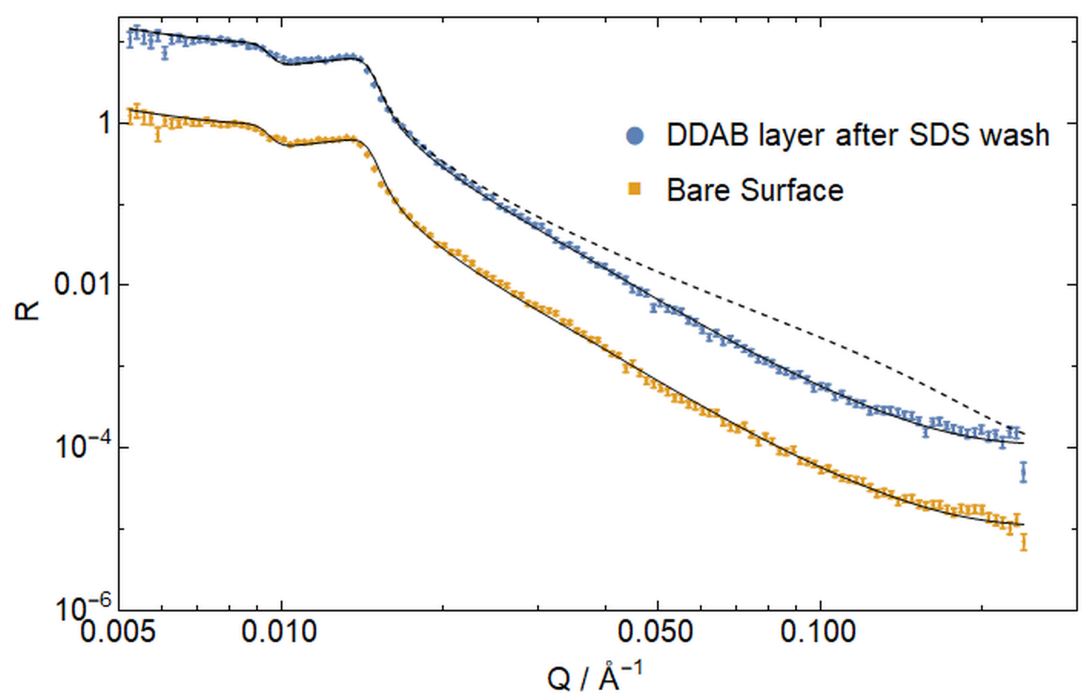

Figure 4: Neutron reflectivity data from the bare mica substrate in $\mathrm{D}_{2} \mathrm{O}$ before any solutions were introduced (lower profile), and from the mica with DDAB layer after the introduction of 1 CMC ( $8.2 \mathrm{mM})$ SDS which is offset by a factor of 10 (upper profile). Data are offset for clarity but superimpose completely within error. The fits shown to the two datasets are the same, and calculated from the parameters fitted to the bare mica as in Fig. 2 The dashed line shows the fit applied to the DDAB layer after washing with $\mathrm{D}_{2} \mathrm{O}$ but before introduction of SDS, showing clear desorption of the layer.

\subsection{Discussion}

The adsorbed layer of DDAB is significantly thicker at $22 \AA$ than the molecular length, which has been calculated as $16.7 \AA$ (Browning et al. 2014), but 
less than twice this value. The layer has hence been characterised as an interdigitated and/or tilted bilayer. The structure of the bilayer has been analysed in more detail by others (Browning et al. 2014), and is not the focus of this work. The slight decrease in layer thickness after washing without any increase in hydration is ascribed to a slight reordering of the layer and perhaps removal of a small proportion of the surfactant molecules, but there is only a very minor change in the layer.

With the cationic DDAB molecules liberated from the mica surface, charge compensation of the anionic mica surface is still required. The potassium cations initially bound to the freshly cleaved mica surface were replaced by the DDAB layer and will no longer be present in the sample cell after the DDAB, $\mathrm{D}_{2} \mathrm{O}$, and SDS washes through the cell. We therefore consider that the only available cations will be the $\mathrm{Na}^{+}$counter-ions from the SDS solution.

$\mathrm{Na}^{+}$is a strongly hydrated ion which has been observed to bind as an outer sphere complex on mica (Lee et al., 2012) compared to inner sphere adsorption of the native $\mathrm{K}^{+}$. It is not expected to bind as strongly to mica, or other clay minerals, as the native $\mathrm{K}^{+}$(Underwood et al. 2016) or the quaternary ammonium ions which formed the bilayer.

The DDAB bilayer formed on mica has shown to be persistent to washes with a solution of the divalent $\mathrm{Ca}^{2+}$ salt at $10 \mathrm{mM}$, which might suggest that the DDAB has a more favourable interaction with the mica than the $\mathrm{Ca}^{2+}$ ion. The observation that DDAB is removed from mica by SDS micelles at the lower concentration of $8.2 \mathrm{mM}$, when the only ion available to compensate the surface charge is $\mathrm{Na}^{+}$, suggests that the favourable energetic interaction of incorporating the DDAB molecules into the anionic SDS micelles is greater than the difference in strength of interaction between the mica - DDAB and mica - $\mathrm{Na}^{+}$. The inclusion of cationic surfactant molecules into anionic micelles is expected to be significantly favourable, as there is both an attractive interaction between the oppositely charged head groups and a reduced repulsion between the like-charged head groups now separated by the included cationic surfactant and we conclude that this driving force is sufficient to result in DDAB removal. 
Using the area per molecule of DDAB in the adsorbed layer $\left(32 \AA^{2}\right.$ as calculated from the molecular volume and layer thickness), the dimensions of the mica - liquid interface in the cell $(40 \mathrm{~mm} \times 85 \mathrm{~mm})$, and the cell volume $(2$ ml) which was the volume of SDS solution in 'initial' contact with the DDAB layer, a very rough estimate of the concentration ratio of DDAB to SDS can be calculated when the SDS is introduced to the cell. The concentration of DDAB in this solution was calculated as $9 \times 10^{-3} \mathrm{mM}$, three orders of magnitude lower than the SDS in solution $(8.2 \mathrm{mM})$. The molar ratio is then $1 \times 10^{-3}$, well below the minimum value for precipitation of 0.06 proposed by Marques et al. (1999). The estimate of the concentration ratio is very approximate and the local ratio at the DDAB layer surface will be higher before the layer de-adsorbs, but no precipitation was observed either in the waste solution leaving the cell or inside the cell after disassembly.

We plan additional studies to observe how an adsorbed layer of DDAB behaves in contact with solutions of SDS below the CMC value of $8.2 \mathrm{mM}$. This will probe whether micelles of anionic surfactant are required to favour the DDAB moving into the bulk and away from the surface, or whether interaction with anionic surfactants in solution but not in aggregates is sufficient to remove or partially remove the adsorbed layer. Investigation of other surfactant systems is also planned.

In many organoclays a cationic organic is used to modify the interlayer or outer surfaces of a clay. In some cases DDAB is the specific organic modifier (Undabeytia et al. 2008). The molecules in the interlayer, rather than the exposed basal plane investigated in this case, may be less accessible to anionic surfactants in solution but the results reported here suggest that consideration should be given to the potential effect of anionic surfactants on the stability of these systems. The organoclays are generally synthesised from clays with a lower layer charge than mica which could result in a less strongly bound organic layer and hence more potential for removal. 


\section{Conclusions}

Through the use of NR onto the mica basal plane, the behaviour of an adsorbed DDAB bilayer has been investigated. The layer was observed to be strongly bound and essentially unperturbed by an extended water wash but, despite the lack of obvious cationic species to compensate the mica surface charge, complete desorption was observed when the surface was exposed to SDS solution at the $\mathrm{CMC}$ value $(8.2 \mathrm{mM})$. This unexpected result has implications both for the use of surfactants in oil recovery, and other avenues of clay science such as organoclays.

\section{Acknowledgements}

We thank BP PLC for funding (RG8620), and specifically Stephanie Houston and Isabella Stocker for their input. We also thank ISIS (RB 1710119, DOI:10.5286/ISIS.E.86389514) and the beamtime committee for the allocation of beamtime, as well as the beamline scientists for their invaluable support.

\section{References}

Allen, F.J., Griffin, L., Alloway, R.M., Gutfreund, P., Lee, S.Y., Truscott, C.L., Welbourn, R.J.L., Wood, M.H., Clarke, S.M., 2017. Anionic Surfactants on Anionic Substrate: Monovalent Cation Binding. Langmuir 33, 7881-8. URL: http://pubs.acs.org/doi/pdf/10.1021/acs.langmuir. 7b01837http://pubs.acs.org/doi/abs/10.1021/acs.langmuir.7b01837, doi:10.1021/acs.langmuir.7b01837.

Blom, A., Warr, G.G., Nelson, A., 2007. Structure of mixed DTAB/DDAB adsorbed layers on quartz. Colloids and Surfaces A: Physicochemical and Engineering Aspects 310, 1-8. URL: http://ac. els-cdn.com/S0927775707005791/1-s2.0-S0927775707005791-main. pdf?\{_\}tid=c8876984-7130-11e7-8f35-00000aacb35d $\{\&\}$ acdnat $=$ 1500984180\{_\}baf235e37128fb6a7d22381f5ba36bb2http://linkinghub. 
elsevier.com/retrieve/pii/S0927775707005791\{\%\}5Cnpapers2:

//publication/d, doi:10.1016/j.colsurfa.2007.05.052.

Browning, K.L., Griffin, L.R., Gutfreund, P., Barker, R.D., Clifton, L.A., Hughes, A., Clarke, S.M., 2014. Specular neutron reflection at the mica/water interface - Irreversible adsorption of a cationic dichain surfactant. Journal of Applied Crystallography 47, 1638-46. doi 10.1107/S1600576714016318.

Dubois, M., Zemb, T., 1991. Phase behavior and scattering of double-chain surfactants in diluted aqueous solutions. Langmuir 7, 1352-60. URL: http://pubs.acs.org/doi/pdf/10.1021/la00055a011http://apps . webofknowledge.com/full\{_\}record.do?product=UA\{\&\}search\{_\}mode=

GeneralSearch $\{\&\} q i d=1\{\&\}$ SID=1FG1SlAqMvIt8Gkijbx $\{\&\}$ page $=1\{\&\}$ doc $=$ 1. doi:10.1021/la00055a011.

Griffin, L.R., Browning, K.L., Truscott, C.L., Clifton, L.a., Clarke, S.M., 2015. Complete Bilayer Adsorption of C16TAB on the Surface of Mica Using Neutron Reflection. The Journal of Physical Chemistry B 119, 64576461. URL:http://www.ncbi.nlm.nih.gov/pubmed/25942291, doi 10 . $1021 /$ acs . jpcb.5b00397

Griffin, L.R., Browning, K.L., Truscott, C.L., Clifton, L.A., Webster, J., Clarke, S.M., 2016. A comparison of didodecyldimethylammonium bromide adsorbed at mica/water and silica/water interfaces using neutron reflection. Journal of Colloid and Interface Science 478, 365-73. doi:10.1016/j.jcis.2016.06. 015.

Grillo, I., Penfold, J., Tucker, I., Cousin, F., 2009. Spontaneous formation of nanovesicles in mixtures of nonionic and dialkyl chain cationic surfactants studied by surface tension and SANS. Langmuir 25, 3932-43. URL: http: //pubs.acs.org/doi/pdf/10.1021/la802435h, doi 10.1021/la802435h.

Holland, P., Rubingh, D., 1983. Nonideal Multicomponent Mixed Micelle Model. 1. The Journal of Physical Chemistry 87, 1984-90. URL: http://pubs.acs. org/doi/pdf/10.1021/j100234a030, doi:10.1021/j100234a030. 
Kaler, E.W., Herrington, K.L., Murthy, A.K., Zasadzinski, J.A.N., 1992. Phase behavior and structures of mixtures of anionic and cationic surfactants. The Journal of Physical Chemistry 96, 6698-707. URL: http://pubs.acs.org/ doi/abs/10.1021/j100195a033, doi:10.1021/j100195a033

Lee, S.S., Fenter, P., Nagy, K.L., Sturchio, N.C., 2012. Monovalent ion adsorption at the muscovite (001)-solution interface: Relationships among ion coverage and speciation, interfacial water structure, and substrate relaxation. Langmuir 28, 8637-50. URL: http://pubs.acs.org/doi/pdf/10. 1021/la300032h doi $10.1021 / 1 \mathrm{a} 300032 \mathrm{~h}$

Marques, E., Khan, A., Miguel, M.D.G., Lindman, B., 1993. Self-Assembly in Mixtures of a Cationic and an Anionic Surfactant : The Sodium Dodecyl Sulfate-Didodecyldimethylammonium Bromide- Water System. Journal of Physical Chemistry 97, 4729-36. URL: http://pubs.acs.org/doi/pdf/10. 1021/j100120a028, doi $10.1021 / \mathrm{j} 100120 \mathrm{a} 028$

Marques, E.F., Regev, O., Khan, A., da Graça Miguel, M., Lindman, B., 1999. Vesicle Formation and General Phase Behavior in the Catanionic Mixture SDSDDABWater. The Anionic-Rich Side. The Journal of Physical Chemistry B 102, 6746-58. URL: http://pubs.acs.org/doi/abs/10. 1021/jp990852p, doi:10.1021/jp990852p.

Mukerjee, P., Mysels, K.J., 1971. Critical Micelle Concentration of Aqueous Surfactant Systems. Technical Report. US Department of Commerce, National Bureau of Standards. Washington, DC.

de Paiva, L.B., Morales, A.R., Valenzuela Díaz, F.R., 2008. Organoclays: Properties, preparation and applications. Ap1 plied Clay Science 42, 8-24. URL: http://ac.els-cdn. com/S0169131708000495/1-s2.0-S0169131708000495-main.pdf?

\{_\}tid=a3137c04-6e08-11e7-857d-00000aacb361 $\{\&\}$ acdnat $=$

$1500637083\left\{\_\right.$fa69f697f1c5c4d85bbd34140c4d9205 doi $10.1016 / j$. clay.2008.02.006. 
Perkin, S., 2012. Ionic liquids in confined geometries. Physical Chemistry Chemical Physics 14, 5052. URL: http://xlink.rsc.org/?DOI=c2cp23814d doi:10.1039/c2cp23814d

Schlegel, M.L., Nagy, K.L., Fenter, P., Cheng, L., Sturchio, N.C., Jacobsen, S.D., 2006. Cation sorption on the muscovite $\left(\begin{array}{lll}0 & 0 & 1\end{array}\right)$ surface in chloride solutions using high-resolution X-ray reflectivity. Geochimica et Cosmochimica Acta 70, 3549-65. URL: http://ac. els-cdn.com/S0016703706001906/1-s2.0-S0016703706001906-main. pdf?\{_\}tid=d3795006-7053-11e7-aa0a-00000aab0f02\{\&\}acdnat $=$

1500889279\{_\}a971bc488b75b3c921a6466c82323c35 doi $10.1016 / j$. gca.2006.04.011.

Sheng, J.J., 2015. Status of surfactant EOR technology. Petroleum 1, 97-105. URL: http://linkinghub.elsevier.com/retrieve/pii/ S2405656115000334, doi $10.1016 / \mathrm{j}$.petlm.2015.07.003.

Sivia, D.S., 2011. Elementary scattering theory : for X-ray q and neutron users. Oxford University Press. URL: https: a //books.google.co.uk/books?id=j4IKOazVdnMC $\{\&\}$ pg=PA193\{\&\}lpg= PA193 $\{\&\} d q=d+$ s+siviatelementary+scattering $+r e f$ erence $\{\&\}$ source $=$

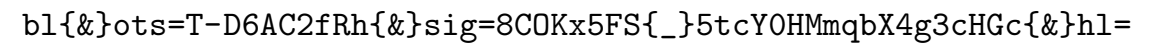
en $\{\&\}$ sa $=X\{\&\}$ ved=0ahUKEwjCmL6V5abVAhUIYVAKHSsNBMcQ6AEIVTAI $\{\#\}$ v= onepage $\{\&\} q=$ dssiviaelemen.

Sohrabi, B., Gharibi, H., Tajik, B., Javadian, S., Hashemianzadeh, M., 2008. Molecular interactions of cationic and anionic surfactants in mixed monolayers and aggregates. Journal of Physical Chemistry B 112, 14869-76. URL: http: //pubs.acs.org/doi/pdf/10.1021/jp803105n doi 10.1021/jp803105n.

Speranza, F., Pilkington, G.A., Dane, T.G., Cresswell, P.T., Li, P., Jacobs, R.M.J., Arnold, T., Bouchenoire, L., Thomas, R.K., Briscoe, W.H., 2013. Quiescent bilayers at the micawater interface. Soft Matter 9, 7028. URL: http://xlink.rsc.org/?DOI=c3sm50336d, doi:10.1039/c3sm50336d. 
Striolo, A., Grady, B.P., 2017. Surfactant Assemblies on Selected NanoStructured Surfaces : Evidence, Driving Forces, and Applications.

n Langmuir URL: http://pubs.acs.org/doi/pdf/10.1021/acs.langmuir. 7b00756, doi:10.1021/acs.langmuir.7b00756

Undabeytia, T., Nir, S., Sánchez-Verdejo, T., Villaverde, J., Maqueda, C., Morillo, E., 2008. A clay-vesicle system for water purification from organic pollutants. Water Research 42, 1211-9. doi:10.1016/j.watres.2007.09.004

Underwood, T., Erastova, V., Chris Greenwell, H., 2016. Ion adsorption at ClayMineral surfaces: The hofmeister series for hydrated smectite minerals. Clays and Clay Minerals 64, 472-87. URL: http://www.ingentaconnect.com/ content/10.1346/CCMN.2016.0640310, doi:10.1346/CCMN.2016.0640310.

Webster, J., Holt, S., Dalgliesh, R., 2006. INTER the chemical interfaces reflectometer on target station 2 at ISIS. Physica B: Condensed Matter 385-386, 1164-6. doi $10.1016 / \mathrm{j} \cdot$ physb.2006.05.400.

Zhao, H., Bhattacharjee, S., Chow, R., Wallace, D., Masliyah, J.H., Xu, Z., 2008. Probing surface charge potentials of clay basal planes and edges by direct force measurements. Langmuir 24, 12899-910. URL: http://pubs . acs.org/doi/pdf/10.1021/la802112h doi 10.1021/la802112h. 